Article

\title{
An Ensemble-Based Approach to Anomaly Detection in Marine Engine Sensor Streams for Efficient Condition Monitoring and Analysis
}

\author{
Donghyun Kim ${ }^{1}$, Sangbong Lee ${ }^{2}$ and Jihwan Lee ${ }^{3, * \mathbb{D}}$ \\ 1 Korea Marine Equipment Research Institute, Busan 49111, Korea; kimdonghyun9942@gmail.com \\ 2 Lab021 Shipping Analytics, Busan 48508, Korea; sblee@lab021.co.kr \\ 3 Department of Industrial Data Engineering, Industrial Data Science and Engineering, \\ Pukyong National University, Busan 48513, Korea \\ * Correspondence: jihwan@pknu.ac.kr; Tel.: +82-51-629-6492
}

Received: 22 November 2020; Accepted: 16 December 2020; Published: 18 December 2020

\begin{abstract}
This study proposes an unsupervised anomaly detection method using sensor streams from the marine engine to detect the anomalous system behavior, which may be a possible sign of system failure. Previous works on marine engine anomaly detection proposed a clustering-based or statistical control chart-based approach that is unstable according to the choice of hyperparameters, or cannot fit well to the high-dimensional dataset. As a remedy to this limitation, this study adopts an ensemble-based approach to anomaly detection. The idea is to train several anomaly detectors with varying hyperparameters in parallel and then combine its result in the anomaly detection phase. Because the anomaly is detected by the combination of different detectors, it is robust to the choice of hyperparameters without loss of accuracy. To demonstrate our methodology, an actual dataset obtained from a 200,000-ton cargo vessel from a Korean shipping company that uses two-stroke diesel engine is analyzed. As a result, anomalies were successfully detected from the high-dimensional and large-scale dataset. After detecting the anomaly, clustering analysis was conducted to the anomalous observation to examine anomaly patterns. By investigating each cluster's feature distribution, several common patterns of abnormal behavior were successfully visualized. Although we analyzed the data from two-stroke diesel engine, our method can be applied to various types of marine engine.
\end{abstract}

Keywords: marine engine; two-stroke diesel engine; onboard sensor; condition monitoring; unsupervised anomaly detection; ensemble learning; clustering analysis; anomaly analysis

\section{Introduction}

The main engine is the most important subsystem that provides the propulsion power of the vessel. Because the failure of the engine during the operation may cause a tremendous economic loss [1], the maintenance of the engine is considered as a critical activity, not for the maintenance routine, but for the vessel classification, which is a process that verifies equipment against a set of technical standards [2]. In maritime industry, the standard practice of engine maintenance follows Planned Maintenance System (PMS), where the machinery is replaced at predetermined time intervals or operating hours, regardless of its actual status [3]. From the economic point of view, however, PMS may not be an optimal strategy because it may have an unnecessary substitution of the machinery.

An alternative strategy to PMS is Conditioned-based Maintenance (CM), wherein the maintenance is carried out based on the condition of the machinery, which is detected by measuring several parameters during the vessel operation [4]. Fortunately, the recent development of IT technology has enabled real-time access to the machinery's condition and energy efficiency using data collected from 
onboard sensors [5-13]. Such sensor-based monitoring can be used to detect abnormal behavior of the system that may indicate the degradation or fault of the system. This study proposes a data-driven approach that enables conditioned-based monitoring of the vessel's main engine utilizing a machine learning algorithm.

We adopt an unsupervised approach in the detection of anomalous system behavior. Although most industries, including the maritime industry, continuously collect data from sensors, most of the data usually comes from the normal operating condition, and a comprehensive fault dataset is usually hard to obtain. Thus, anomaly detected from our methodology may not be directly related to the system fault because any behavior that shows a large deviation from the normal dataset can be detected as an anomaly. However, they could be used for the initial screening of engine status monitoring and can be combined with further analysis, including fault isolation and diagnosis.

Previously, several unsupervised methods have been proposed to analyze onboard sensor data in anomaly detection for the marine engine. The clustering-based approach adopts a clustering algorithm to identify clusters in the sensor data first and then check whether new data belongs to existing clusters. They assume that normal instances have stronger adherence to clusters than the anomaly. Perera and Mo $[14,15]$ classified the most frequent operating regions of the marine engine. In their work, the Gaussian Mixture Model is adopted to represent the cluster of operating regions as a mixture of probability distributions. Brandsæter and Venem [16] proposes an efficient online method to calculate the degree of abnormality from clusters. Vanem and Brandsæter [17] compare several clustering algorithms by analyzing anomaly detection results. Although the Clustering-based approaches are intuitive and easy-to-implement, it suffers from unstable because the clustering result may be largely affected by the number of clusters, which should be specified by the user. Although there are several guidelines for determining the good cluster numbers, they cannot be applicable to the unsupervised dataset. As an alternative approach, Bae et al. [18] proposed a Statistical Process Control (SPC)-based approach to the anomaly detection of the vessel engine. To remove the distributional assumption of the dataset, a Bootstrap-based T2 multivariate chart proposed by Phaladiganon et al. [19], is adopted to determine the threshold for each sensor data. If one of the sensor values is out of the threshold, the data point is detected as an outlier. However, SPC-based approach suffers from low performance when the dataset involves high dimensional spaces [20].

To overcome the limitation of the above approaches, we propose an ensemble-based anomaly detection method to operate on a large-scale high-dimensional dataset. The idea is to apply several algorithms with varying hyperparameters to the same dataset and then combine each classifier's anomaly detection result. Because the anomaly is determined by the combination of multiple classifiers, the result is robust to the choice of hyperparameters without loss of its prediction power. To demonstrate our methodology, a data stream obtained from a 200,000-ton bulk cargo ship operated by a Korean shipping company that is collected during ten months are analyzed. The data set consists of comprehensive parameters representing engine performance, including engine rotation per minute (RPM), temperature, and pressures of lubricant oil and cooling waters. Several preprocessing steps were conducted to reduce the data size and select the informative sensor parameters. The ensemble-based algorithm then trains the preprocessed data to detect the anomalies from the input data. After detecting the anomaly, clustering analysis was conducted to the anomalous observation to examine anomaly patterns. By investigating each cluster's feature distribution, several common patterns of abnormal behavior were successfully visualized. The result shows that the proposed method can be successfully applied to the large and high-dimensional sensor streams. Although we analyzed the data from two-stroke diesel engine, our method can be applied to various types of marine engine.

The remainder of this paper is organized as follows. Section 2 explains about the target vessel and data set collected from the vessel. Section 3 addresses the procedures used for data preprocessing. Section 4 illustrates the ensemble-based machine learning model used for anomaly detection of the engine status. In Section 5, an in-depth discussion about anomaly detection results was performed. Finally, Section 6 addresses the limitation and future work of this study. 


\section{Data Description}

This section explains the description of our target vessel and the dataset used in anomaly detection. The target vessel is a 200,000-ton bulk cargo ship, and its detailed specification is in Table 1 . The data collection period spans about ten months, starting from 2019 July to 2020 April. As shown in Figure 1, its routes include main ports in Asian countries including Korea, Russia, Singapore, and Taiwan. The sensor measured the data at a one-second interval, resulting in a total of 22,513,800 observations.

Table 1. Specification of the target vessel.

\begin{tabular}{cc}
\hline Specification & \\
\hline Length Overall & $269.36 \mathrm{~m}$ \\
Length between perpendiculars & $259.00 \mathrm{~m}$ \\
Breadth & $43.00 \mathrm{~m}$ \\
Depth & $23.80 \mathrm{~m}$ \\
Draught & $17.3 \mathrm{~m}$ \\
Deadweight & 152.517 metric t \\
\hline
\end{tabular}

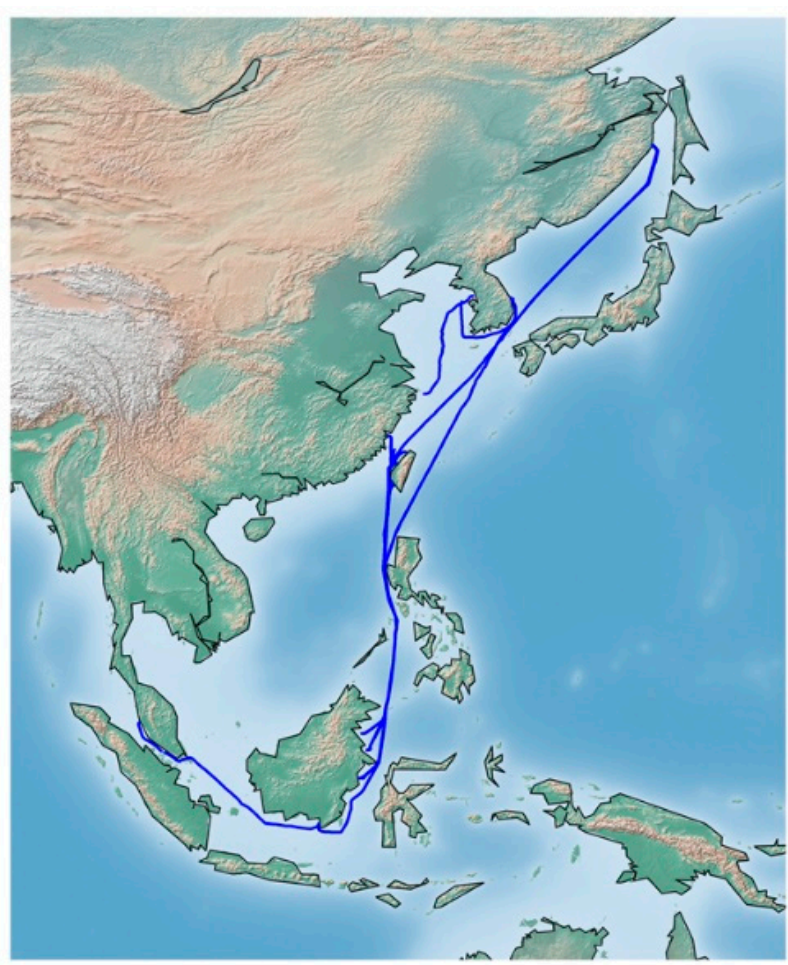

Figure 1. Vessel operation routes over the data collection period.

The engine model used in the vessel is MAN B\&W MC50, which is a slow-speed two-stroke engine [21]. The engine adopted Variable Injection Timing (VIT) systems that control the timing of the start of the fuel injection. The coolant system uses lubricant cooling for the rotating part (Crankshaft, Piston), and the fixed part (Cylinder Head, Jacket) is cooled with fresh water. The coolant is cooled by seawater in a separate heat exchanger.

In the raw dataset, more than 150 data streams were collected by onboard sensors. Some parameters were related with navigational information such as Global Positioning System (GPS) location, ground speed, wind speed, water level, etc. In contrast, others were related with subsystems' status, including engine, generator, thruster, and cargo management system, etc. In this study, we only included parameters that are attached on main engine subsystems. Other parameters that come from other subsystems were excluded because they are not in our interest. As a result, the chosen parameters are shown in Table 2. 
Table 2. Description of parameters.

\begin{tabular}{cl}
\hline \multicolumn{1}{c}{ Sensor Name } & \multicolumn{1}{c}{ Description } \\
\hline ME1 FO FLOW HOUR INLET & The consumption rate of fuel oil \\
ME1 FO TOTALIZER INLET & Cumulative consumption of fuel oil \\
ME1 FO TEMP INLET & The temperature of fuel oil \\
ME1 FO DENSITY INLET & The density of fuel oil \\
\hline ME1 RPM ECC & Engine rotation per minute (RPM) \\
ME1 RPM & Same as above \\
\hline ME1 SCAV AIR PRESS ECC & The Pressure of scavenging air \\
ME1 SCAV AIR PREE & Same as above \\
ME1 FO INLET TEMP & The inlet temperature of fuel oil \\
ME1 FO INLET PRESS & Inlet pressure of fuel oil \\
\hline
\end{tabular}

ME1 CYL1 PCO OUTLET TEMP

ME1 CYL2 PCO OUTLET TEMP

ME1 CYL3 PCO OUTLET TEMP

ME1 CYL4 PCO OUTLET TEMP

The outlet temperature of cylinder piston cooling oil

ME1 CYL5 PCO OUTLET TEMP

\begin{tabular}{cl}
\hline ME1 JCW INLET TEMP & The inlet temperature of jacket cooling water \\
ME1 JCW INLET OUTLET & The outlet temperature of jacket cooling water \\
ME1 CYL1 CFW OUT TEMP & \\
ME1 CYL3 CFW OUT TEMP & The outlet temperature of cylinder block cooling water \\
ME1 CYL4 CFW OUT TEMP & \\
ME1 CYL5 CFW OUT TEMP & \\
ME1 CYL1 EXH GAS OUTLET TEMP & \\
ME1 CYL2 EXH GAS OUTLET TEMP & \\
ME1 CYL3 EXH GAS OUTLET TEMP & The outlet temperature of exhaust gas \\
ME1 CYL5 EXH GAS OUTLET TEMP & \\
\hline ME1 TC1 EXH INLET TEMP & The inlet temperature of exhaust gas of turbocharger \\
ME1 TC1 EXH OUTLET TEMP & The outlet temperature of exhaust gas of turbocharger \\
ME1 TC LO OUTLET TEMP & The outlet temperature of lubricant oil \\
\hline ME1 LO INLET PRESS & Inlet pressure of lubricant oil \\
ME1 LO INLET TEMP & The inlet temperature of lubricant oil \\
\hline
\end{tabular}

\section{Data Preprocessing}

All the data-driven approach requires representative training dataset. However, the raw data stream is not complete. It may contain out-of-range values, missing values, redundant variables, and irreverent information. If the raw data is not carefully screened, then the resulting model will not perform well on the new data. Thus, in this study, several preprocessing methods were applied to improve the quality of the dataset. The overall analysis framework is shown in Figure 2.

First, out-of-range values that exceed the acceptable sensor value ranges were removed. In some cases, sensor value shows zero or extremely large values that are out of acceptable range of sensors. Those values are usually the consequence of signal loss either from the sensors or from the communication. Because both cases were not related to the failure of the engine, it is natural to remove such outliers in the training data set.

Then, we reduce the dataset by averaging its value with a 10-min interval. One reason for this transformation is that the current data set (measured with a second interval) is too huge to train the model. Besides, the vessel engine usually undergoes slow changes during the operation compared to other vehicles such as the car or the airplane. Thus, averaging the dataset with a 10-min interval may be enough for training the model. As a result, the size of the dataset was reduced to 37,523. 


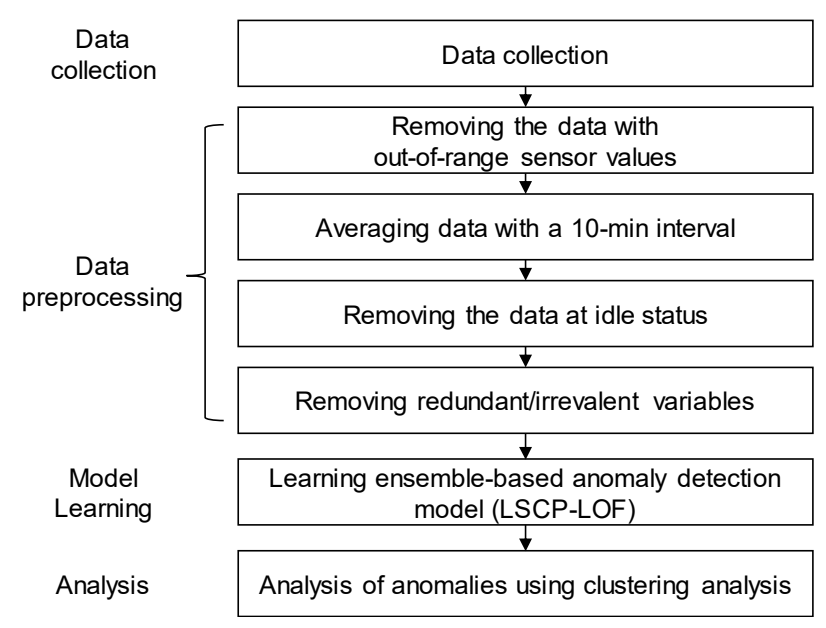

Figure 2. The overall framework for vessel main engine anomaly detection.

Next, we exclude the data collected when the vessel was idle because the vessel engine does not operate during that period. As shown in Figure 3, the vessel shows the alternating operational status (idle and normal operation) during the data collection period. This study adopts a window-based change point detection algorithm to the ground speed time-series data to distinguish the vessel's operational status. The algorithm tries to detect the rapid change points using two windows, which slide along the data stream. The statistical properties of each window are compared with a discrepancy measure. For a given cost function $c(\cdot)$, a discrepancy measure $d(\cdot, \cdot)$ as follow:

$$
d\left(y_{u, v}, y_{v, w}\right)=c\left(y_{u, w}\right)-c\left(y_{u, v}\right)-c\left(y_{v, w}\right)
$$

where $y_{t}$ is the input time series at time point $t$ and $u<v<w$ are indexes. If the discrepancy measure between two sliding windows is smaller, this indicates that there is no change point at v. On the other hand, if the sliding windows fall into two dissimilar segments, the discrepancy is significantly higher, suggesting that $v$ is a change point. In this study, such a change point indicates the boundary between the operational status of the vessel. Because the time window is considered for change point detection, this method is less sensitive to the noise data. For more details about the methods, please refer to [22]. Figure 3 also shows change points detected by the time-window based method. In this study, we consider the area whose average ground speed is over 6 knots. Further, according to an expert opinion, we determined to consider the dataset whose RPM value is over 70 .

Then, feature selection and transformation was conducted. The feature selection result and was summarized in Table 3. As shown in the table, from the original dataset, some parameters (ME1 RPM ECC, ME1 SCAV AIR PRESS ECC) were obtained from duplicated sensors of other sensors (ME1 RPM, ME1 SCAV AIR PRESS) in case of sensor failures. Because parameter values of original and duplicated sensors were exactly same throughout the data collection period, we excluded duplicated sensor parameters from the dataset. In addition, parameters related with the fuel status (ME1 FO FLOW INLET, ME1 FO DESNITY INLET, ME1 FO TEMP INLET, ME1 FO TOTALIZER INLET) were removed. Of course, the change in fuel density or temperature may affect the performance of the engine. Especially, if a vessel sailed through an emission control area, such as the western part of the United States that regulates the use of low sulfur oil, the reduced lubrication effect due to low-sulfur oil might increase the probability of accidents, such as piston sticking. However, because our vessel has not sailed through an emission control area, there was no significant change in fuel oil (such as viscosity) during the data collection period. Moreover, the vessel used fuel additives to prevent the problem that may arise from fuel status. Finally, instead of using individual sensor value of individual cylinder, we use averaged value because sensor values from five cylinders show high correlation with each other as shown in Figure 4. As a result, the variable size was reduced from 32 to 14. 

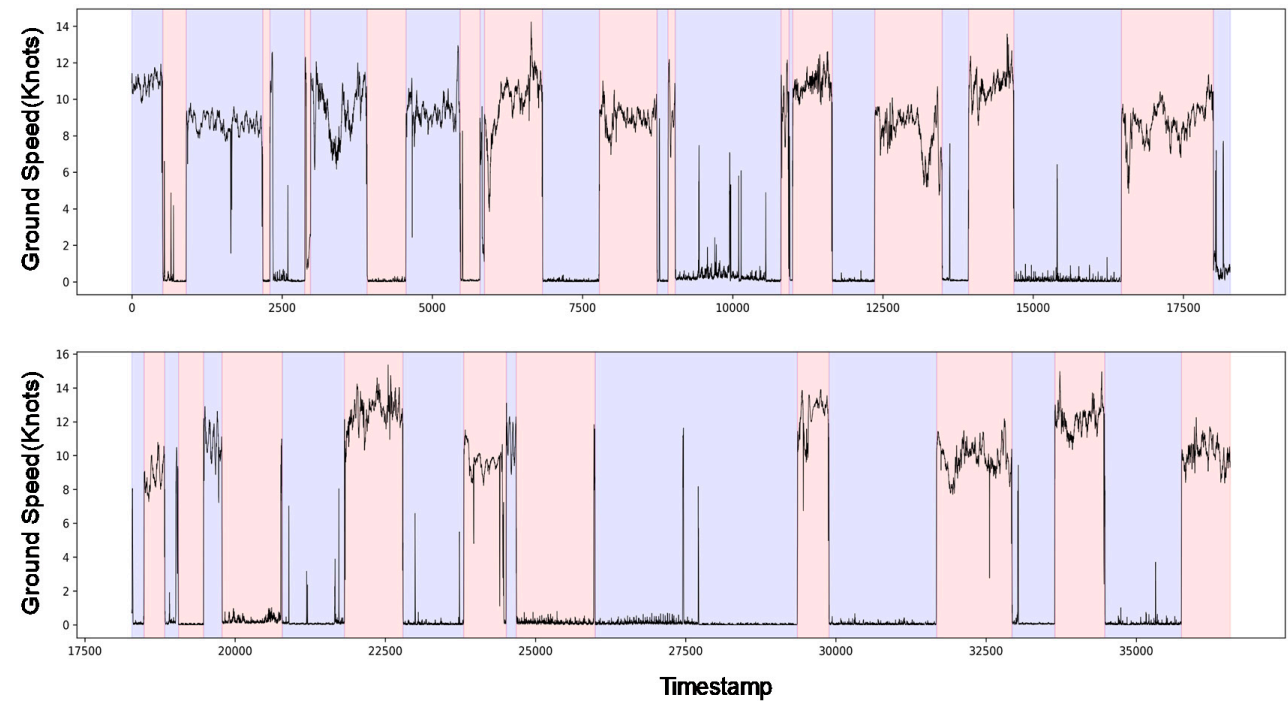

Figure 3. Time series of the vessel ground speed. Moreover, the windows-based change detection method is also applied to find operational regions among the dataset. The alternating color region indicates whether the vessel operates or not.

Table 3. Removed Parameters and Reason.

\begin{tabular}{cc}
\hline Reason & Parameters \\
\hline $\begin{array}{c}\text { Fuel Oil Status Indicator } \\
\text { (not affect engine condition) }\end{array}$ & $\begin{array}{c}\text { ME1 FO FLOW HOUR INLET, ME1 FO DENSITY INLET, } \\
\text { ME1 FO TEMP INLET, ME1 FO TOTALIZER INLE }\end{array}$ \\
\hline Duplicated sensors & ME1 RPM ECC, ME1 SCAV AIR PREES ECC \\
\hline & ME1 [CYL1 CYL5] PCO OUTLET TEMP \\
Aggregate value by averaging & ME1 [CYL1 CYL5] PCO OUTLET TEMP \\
& ME1 [CYL1 CYL5] CFW OUTLET TEMP \\
\hline
\end{tabular}

ME1_FO_FLOW_HOUR_INLET ME1_RPM - $\because 、$ ME1_SCAV_AIR_PRESS ME1_CYL1_PCO_OUTLET_TEMP - V ME1_CYL2_PCO_OUTLET_TEMP ME1_CYL3_PCO_OUTLET_TEMP -..ME1_CYL4_PCO_OUTLET_TEMP -.... ME1_CYL5_PCO_OUTLET_TEMP -... ME1 JCW_INLET_TEMP - - - -

ME1_CW_INLET_PRESS ..... MEI_CYLI_CFW_OUTLET_TEMP ...-ME1_CYL2_CFW_OUTLET_TEMP .., ME1_CYL3_CFW_OUTLET_TEMP ., ME1_CYL4_CFW_OUTLET_TEMP - , ME1_CYL5_CFW_OUTLET_TEMP MEI_CYLI_EXH_GAS_OUTLET_TEMP - $i^{\prime}$, ME1_CYL2_EXH_GAS_OUTLET_TEMP ME1_CYL3_EXH_GAS_OUTLET_TEMP MEI_CYL4_EXH_GAS_OUTLET_TEMP $-1 ;$ ME1_CYL5_EXH_GAS_OUTLET_TEMP $-i_{1}^{\prime}$ ME1_TC1_EXH_INLET_TEMP $i^{\prime}, i$ ME1_TC1_EXH_OUTLET_TEMP ME1_TC1_LO_INLET_PRESS ${ }_{1}^{\prime},{ }^{\prime}$ ME1_TC1_LO_OUTLET_TEMP - ${ }_{1}$, ME1_LO_INLET_PRESS ME1_LO_INLET_TEMP - - '

Figure 4. Correlation heatmap among entire feature set. 
We may use more sophisticated variable reduction techniques such as Principal Component Analysis (PCA) or Independent Component Analysis (ICA) to reduce the variables further. However, we determine to preserve the current variables to utilize them in several analyses after detecting anomalies for examining common patterns and causes for anomalies. As a result, the comparison between dataset and preprocessed dataset is shown in Table 4.

Table 4. Comparison between original dataset and preprocessed dataset.

\begin{tabular}{lll}
\hline \multicolumn{1}{c}{ Original Dataset } & \multicolumn{1}{c}{ Preprocessed Dataset } \\
\hline ME1 FO FLOW HOUR INLET & ME1 RPM \\
ME1 FO TOTALIZER INLET & ME1 SCAV AIR PRESS \\
ME1 FO TEMP INLET & ME1 FO INLET TEMP \\
ME1 FO DENSITY INLET & ME1 FO INLET PRESS \\
ME1 RPM ECC & ME1 CYL PCO OUTLET TEMP \\
ME1 RPM & (Average value of 5 cylinders) \\
ME1 SCAV AIR PRESS ECC & ME1 JCW INLET TEMP \\
ME1 SCAV AIR PRESS & ME1 JCW INLET OUTLET \\
ME1 FO INLET TEMP & ME1 CYL CFW OUT TEMP \\
ME1 FO INLET PRESS & (Average value of 5 cylinders) \\
ME1 [CYL1 CYL5] PCO OUTLET TEMP & ME1 CYL EXH GAS OUTLET TEMP \\
ME1 JCW INLET TEMP & (Average value of 5 cylinders) \\
ME1 JCW INLET OUTLET & ME1 TC1 EXH INLET TEMP \\
ME1 [CYL1 CYL5] CFW OUT TEMP & ME1 TC1 EXH OUTLET TEMP \\
ME1 [CYL1 CYL5] EXH GAS OUTLET TEMP & ME1 TC LO OUTLET TEMP \\
ME1 TC1 EXH INLET TEMP & ME1 TC LO INLET TEMP \\
ME1 TC1 EXH OUTLET TEMP & ME1 TC LO INLET PRESS \\
ME1 TC LO OUTLET TEMP & \\
ME1 LO INLET PRESS & \\
ME1 LO INLET TEMP & \\
\hline \multirow{2}{*}{ Number of Observations } & 22,513,800 & \\
& (one second interval) & \\
\hline
\end{tabular}

\section{Ensemble-Based Method for Anomaly Detection}

This section outlines the anomaly detection algorithm applied to the preprocessed dataset. This study adopts an unsupervised approach because we have no labeled dataset about the failure of the vessel's main engine during the data collection period. The unsupervised approach assumes that all the training data shows the normal condition [23]. Thus, if a new observation shows a large deviation from the training set, it is considered an anomaly. Unsupervised anomaly detection may show poor performance if the distribution of the normal dataset is heavy-tailed, or the normal data point is too centered to mimic the anomalous data point [24]. Moreover, the low-accuracy problem may be more severe when the feature space of the dataset is high-dimensional [23].

To remedy this problem, ensemble learning is also applied to model learning. The ensemble approach, which combines multiple base estimators in anomaly detection, is considered as a strategy to improve the model accuracy and stability because one can reduce the effect of variance on modeling accuracy by running the model multiple times [25]. Ensemble approach can be categorized as model-centric when multiple based estimators of the different hyperparameter are combined to predict anomaly score, while it is categorized as the data-centric when the different derivatives of the dataset are applied to the same model. Several ensemble-based approaches, such as feature bagging, parametric ensemble, and sub samplings, are available.

\subsection{Base Anomaly Detection Algorithm: Local Outlier Factor}

In this study, the Local Outlier Factor (LOF) [26] is applied as a basic anomaly detector. LOF is considered as an instance-based method because it firstly finds a relevant instance of the training data and makes a prediction using the information of these instances. Because this approach does not require the design of generic models, it is often referred to as memory-based methods. LOF [17] and the 
k-nearest neighborhood-based method [27] was its successful implementation. One of the problems in instance-based methods is that the performance of the anomaly detection may be severely affected by the local distributions of the data. LOF addresses this problem by using density information of its neighborhood point. For a given data point $x_{i}$, let $D^{k}\left(x_{i}\right)$ be the distance between $x_{i}$ and its k-nearest neighbor, and $L_{k}\left(x_{i}\right)$ be the set of points within the k-nearest neighbor distance. Then, we calculate the reachability distance between two data points $x_{i}$ and $x_{j} R_{k}\left(x_{i}, x_{j}\right)$ is calculated as follows:

$$
R_{k}\left(x_{i}, x_{j}\right)=\max \left\{\operatorname{dist}\left(x_{i}, x_{j}\right), D^{k}\left(x_{j}\right)\right\}
$$

when $j$ is in a dense region and the $x_{i}$ is far from $x_{j}$, the reachability index will be equal to the true distance. If $j$ is in sparse region, on the other hand, the reachability index will be smoothed out by its k-nearest neighbor distance. In this way, we can calculate the average reachability distance $A R^{k}\left(x_{i}\right)$ of $x_{i}$ by averaging reachability distance of its k-nearest neighborhood points:

$$
A R^{k}\left(x_{i}\right)=M E A N_{j \in L_{k}\left(x_{i}\right)} R_{k}\left(x_{i}, x_{j}\right)
$$

the local outlier factor is the average ratio of $A R^{k}\left(x_{i}\right)$ with respect to its k-nearest neighborhood of $x_{i}$ :

$$
\operatorname{LOF}_{k}\left(x_{i}\right)=\operatorname{MEAN}_{y_{i} \in L_{k}\left(x_{i}\right)} \frac{A R_{k}\left(x_{i}\right)}{A R_{k}\left(x_{j}\right)}
$$

As the LOF algorithm can detect "local" outliers regardless of the data distribution of normal behavior, it has been applied to various applications, including network intrusion detection and process monitoring [28]. Due to the computational complexity of the LOF algorithm, however, its application to large data with high dimension has been limited. This issue can be more critical for real-time application systems.

\subsection{Ensemble-Based Approach to Anomaly Detection: LSCP}

As an ensemble approach to anomaly detection, Locally Selective Combination in Parallel Outlier Ensembles (LSCP), which is proposed by Zhao et al. [29], is adopted. LSCP is proposed to solve the local data problem when the data consists of heterogeneous distribution, thus cannot be represented by the one generic model. The presence of the local data structure, thus, is considered as one of the main causes that lower the performance of the unsupervised anomaly detection algorithm. LSCP tries to solve this problem by identifying local regions obtained from its nearest neighbor and building competitive ensemble detectors for each local region, thus, providing more robust predictions. Moreover, LSCP utilizes a feature bagging strategy to cope with the problem arise in high-dimensional feature space.

LSCP consists of four major steps, as shown in Figure 5. In the first stage, generate pseudo-ground truth labels are generated from the ensemble. Let $X_{\text {train }}$ be training data, and $C=\left\{C_{1}, C_{2}, \ldots, C_{R}\right\}$ be a collection of base detectors with different hyperparameter settings. Moreover, let $O\left(X_{\text {train }}\right)$ be the matrix of the anomaly score $O\left(X_{\text {train }}\right)=\left[C_{1}\left(X_{\text {train }}\right), \ldots, C_{r}\left(X_{\text {train }}\right)\right]$. Then, the pseudo ground truth denoted by the target is obtained by score aggregation of base estimators $C$ as follows:

$$
\text { target }=f\left(O\left(X_{\text {train }}\right)\right)
$$

In the second stage, local region is constructed. Given a data instance to test $x_{j}$, the local region $\psi_{j}$ is defined as its k-nearest neighborhood defined as the follows:

$$
\psi_{j}=\left\{x_{i} \mid x_{i} \in X_{\text {train }}, x_{i} \in L_{k, E N S}\left(x_{j}\right)\right\}
$$

To define the local region $L_{k, E N S}\left(x_{j}\right), t$ groups of $[\mathrm{d} / 2, \mathrm{~d}]$ features are randomly selected and $\mathrm{k}$ nearest data object is identified. $x_{i}$ is included in $L_{k, E N S}\left(x_{j}\right)$ when $x_{i}$ are included in the neighborhood more than $\mathrm{t} / 2$ times. 
In the Third stage, model selection and combination are conducted. For testing instance $x_{j}$, let target $^{\psi_{j}}$ be the pseudo ground target value from its $\mathrm{k}$ nearest neighborhood:

$$
\begin{gathered}
\text { target }_{j}=\left\{\text { target }_{x_{i}} \mid x_{i} \in \psi_{j}\right\} \\
O\left(\psi_{j}\right)=\left[C_{1}\left(\psi_{j}\right), \ldots, C_{r}\left(\psi_{j}\right)\right]
\end{gathered}
$$

Moreover, let $O\left(\psi_{j}\right)$ be the training score matrix retrieved from its anomaly score matrix:

Then, the correlation between each base detector and pseudo ground truth over the local region is calculated. Pearson correlation is applied between target $\psi_{j}$ and $O\left(\psi_{j}\right)$.

In the final step, a histogram of the Pearson correlation score of each detector is constructed, and then binned with $b$ equal intervals. Then, the collection of detectors belonging to the most frequent intervals are kept for the ensemble for the later stage. Finally, selected detectors scores are combined with the average of maximum strategy. In Zhao et al. [29], LSCP shows better performance on many real datasets. LSCP is also considered in this study because our vessel dataset is collected from several heterogeneous routes, indicating the presence of local structures.

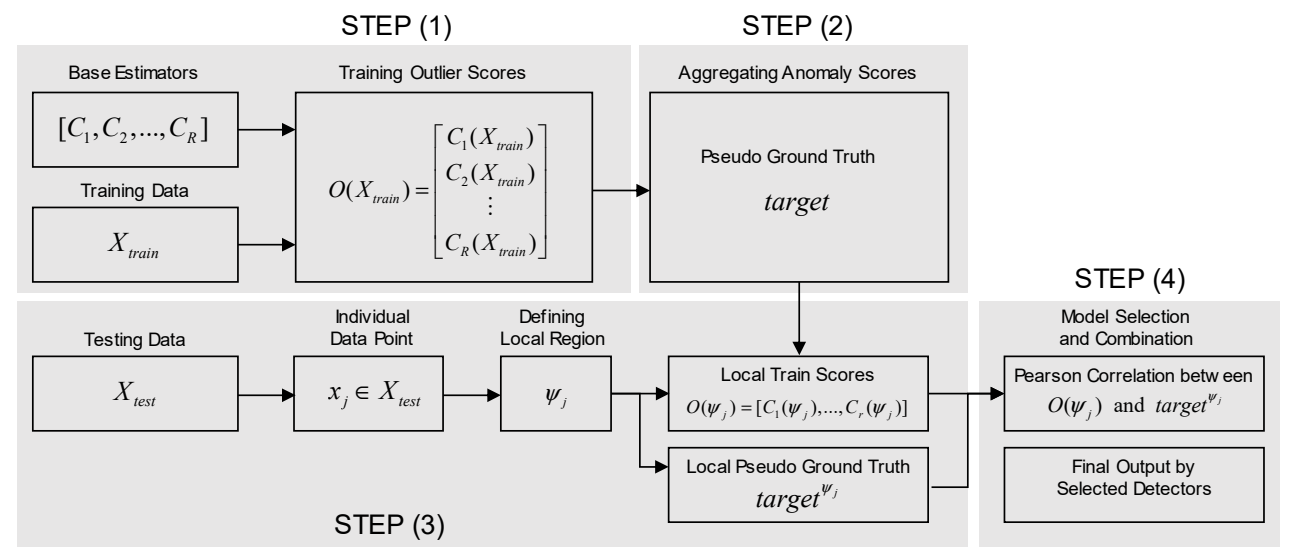

Figure 5. Locally Selective Combination in Parallel Outlier Ensembles (LSCP) Procedure (adopted from Zhao et al. [22]).

\section{Experimental Result}

\subsection{Anomalies Detection Result}

This section illustrates the result of the anomaly detection analysis. To make an ensemble anomaly detector, we combined 30 different LOF detectors. To enhance the robustness of an ensemble detector, it is required to ensure the diversity of base detectors by setting different hyperparameters. In the case of LOF, the dominant hyperparameter is $\mathrm{k}$, which is the number of nearest neighbors to consider. Thus, 30 different hyperparameter set is randomly drawn from integer intervals ranging from 5 to 150 . The numerical experiment was performed on Python 3.6. We used the PYOD (python toolkit for detection of outlying objects) in the implementation of LSCP [30]. The computing environment was CPU 2.2 Ghz, RAM 13 Gb.

Figure 6 shows the histogram of the anomaly scores obtained from the LSCP algorithm. The vertical line indicates the anomaly thresholds with different percentile values. Because the histogram has a very thin tail part, it seems that the anomalous data object is well separated from the normal dataset. As shown in Figure 6, we further highlighted the tail part by limiting the y-axis value to determine the proper threshold. In this study, percentile 0.998 is considered as the threshold because there is a small inflection of histogram, which may suggest the separation between different groups. 


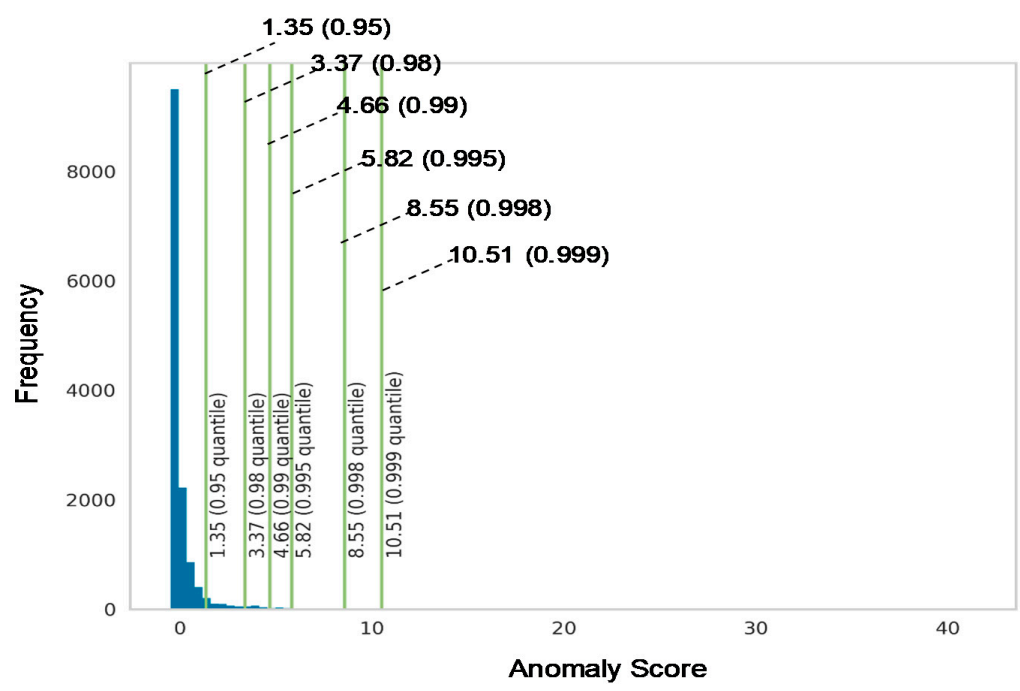

Figure 6. Histogram of anomalies score.

In Figure 7, we compared the anomalies obtained from our ensemble-based model (LSCP) with several individual detectors (LOFs) of varying hyperparameters. As shown in the figure, the anomaly detection result of an individual detector varies according to the hyperparameter. This result suggests that depending only on a single anomaly detector may be biased with the local data structure. On the other hand, anomalies detected from the ensemble-based method seems to be more robust because it includes data points that commonly appear across individual detectors.

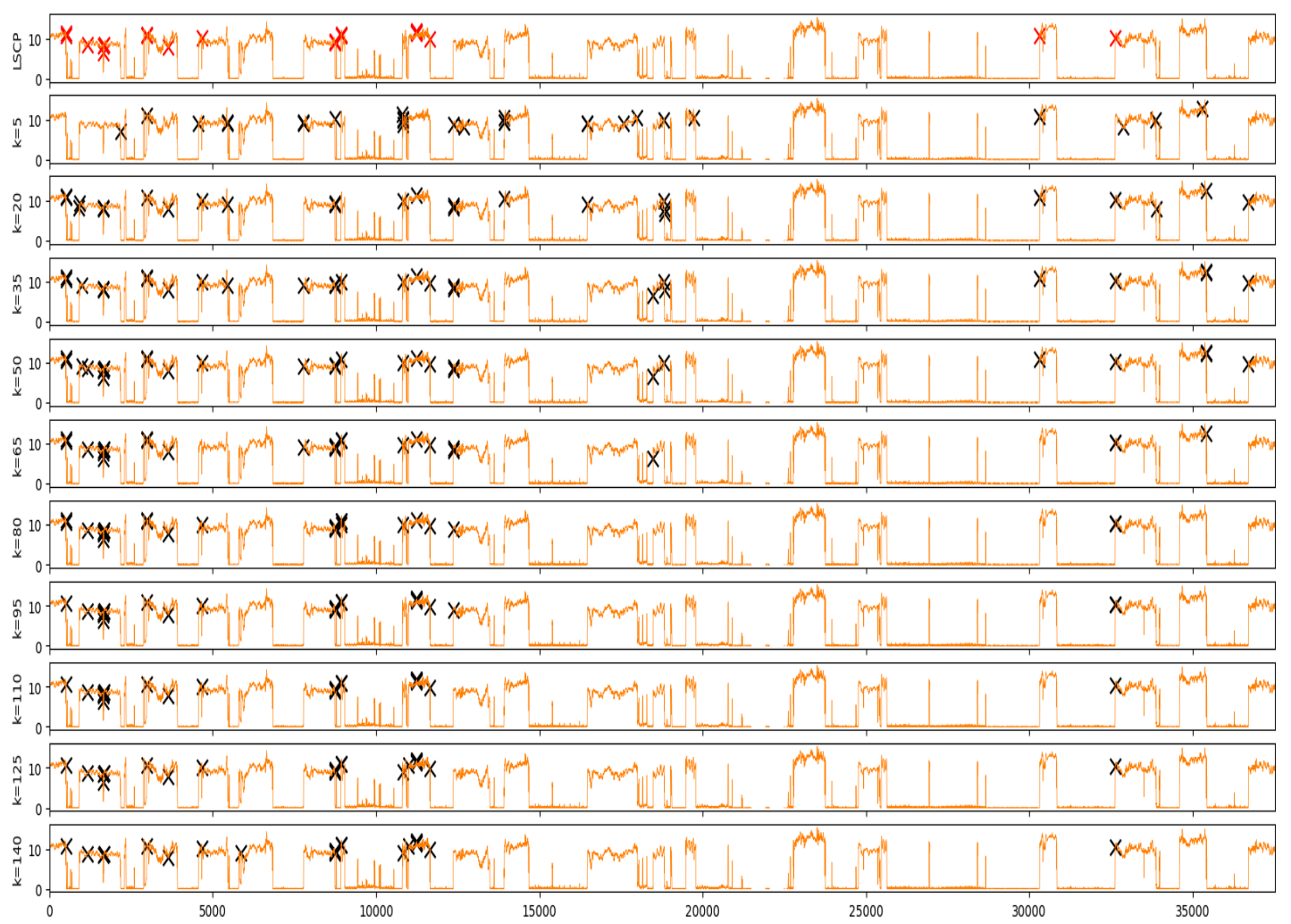

Figure 7. Comparison of anomaly detection results. The red mark indicates anomalies detected by the ensemble-based approach (LSCP), while the black mark indicates anomalies from individual detector Local Outlier Factor (LOF). 


\subsection{Anomalous Pattern Identification Using Clustering Analysis}

We identified clusters of an anomalous dataset to examine the typical patterns of anomalous engine behavior. To this, we applied the K-means algorithm to the anomalous data points detected from our ensemble-based algorithm. As a result, we found four clusters of anomalous engine behavior. Figure 8 compares the distribution of entire variables of each anomalous data cluster. For each sensor variable, we = highlighted the cluster that shows a large deviation from the distribution of normal data points. As shown in the figure, most of the variables were highlighted by cluster 0 (highlighted by the blue line) or cluster 1 (highlighted by the green line). However, cluster 2 and 3 show little distinction from the normal data distribution. Table 5 summarizes the anomalous features associated with each cluster.
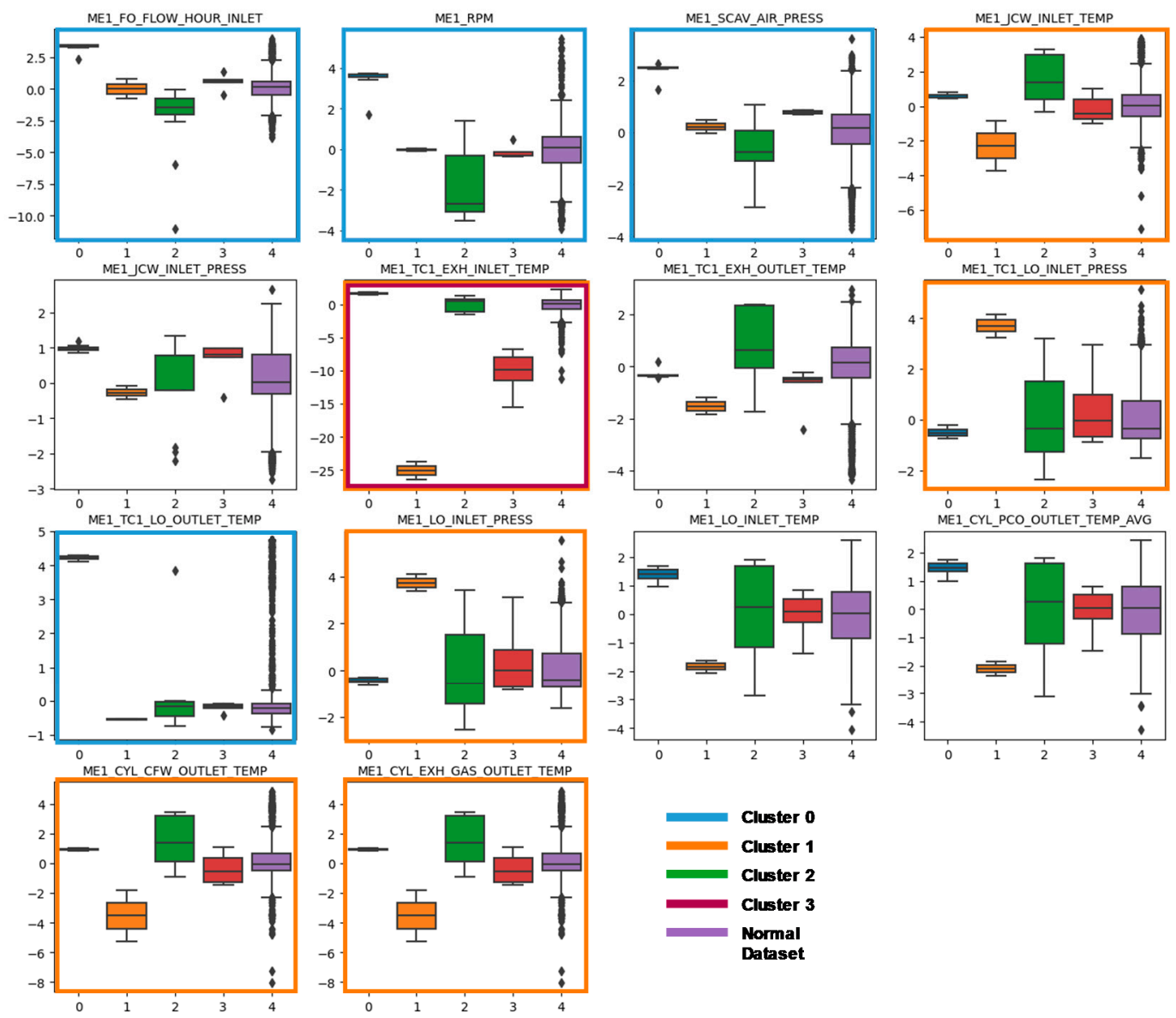

Figure 8. Comparison of boxplots among anomalous data cluster. Clusters that show large deviation from other groups are highlighted by the cluster colors.

Anomalies detected from Cluster 0 show a high value in fuel oil flow rate, RPM, scavenging air pressure, and turbocharger lubricant oil temperature. A possible cause of this anomaly may be the engine's acceleration because all of the relevant parameters seem to be the result caused by the engine acceleration [21]. On the other hand, the possible explanation about the anomalous parameters in cluster 1 seems to engine overcooling of the engine, wherein the normal temperature at which the engine operates cannot be reached. Because the engine overcooling also can damage an engine just as overheating, this region requires further investigation [31]. Cluster 3 is almost the same as cluster 0 except for lower turbocharger exhaust gas temperature. One of the possible causes of this anomaly may be the abnormal intake airflow in a marine engine [32] 
Table 5. Anomalous Parameters of Each Cluster.

\begin{tabular}{cc}
\hline Clusters & Anomalous Features \\
\hline Cluster 0 & High fuel oil flow rate \\
& High engine RPM \\
& High scavenging air pressure \\
& High turbocharger lubricant oil outlet temperature \\
\hline Cluster 1 & Low jacket cooling water inlet temperature \\
& Low turbocharger exhaust gas inlet temperature \\
& High turbocharger lubricant oil inlet pressure \\
& High lubricant oil inlet temperature \\
& Low cylinder block cooling water temperature \\
& Low cylinder exhaust gas outlet temperature \\
\hline Cluster 3 & High scavenging air pressure \\
& Low turbocharger exhaust gas inlet temperature \\
\hline
\end{tabular}

\subsection{Anomalous Engine Status Analysis with Vessel Operational Information}

We conducted several analyses to find some potential causes of the anomalous data point. First, we analyzed the anomalous data point by examining its location on the vessel speed vs. power curve, as shown in Figure 9. Usually, there is a positive relationship between the rpm of the engine and the vessel's ground speed. If most of the anomalous data points have high speed and RPM value, then the engine's load required to operate the vessel at high speed may cause the anomaly. However, as the figure suggests, except for cluster 0 , the anomalous data point in other clusters seems to spread over the speed vs. power curve. This result suggests that our anomalous data points may not have a single cause and calls for further investigation.

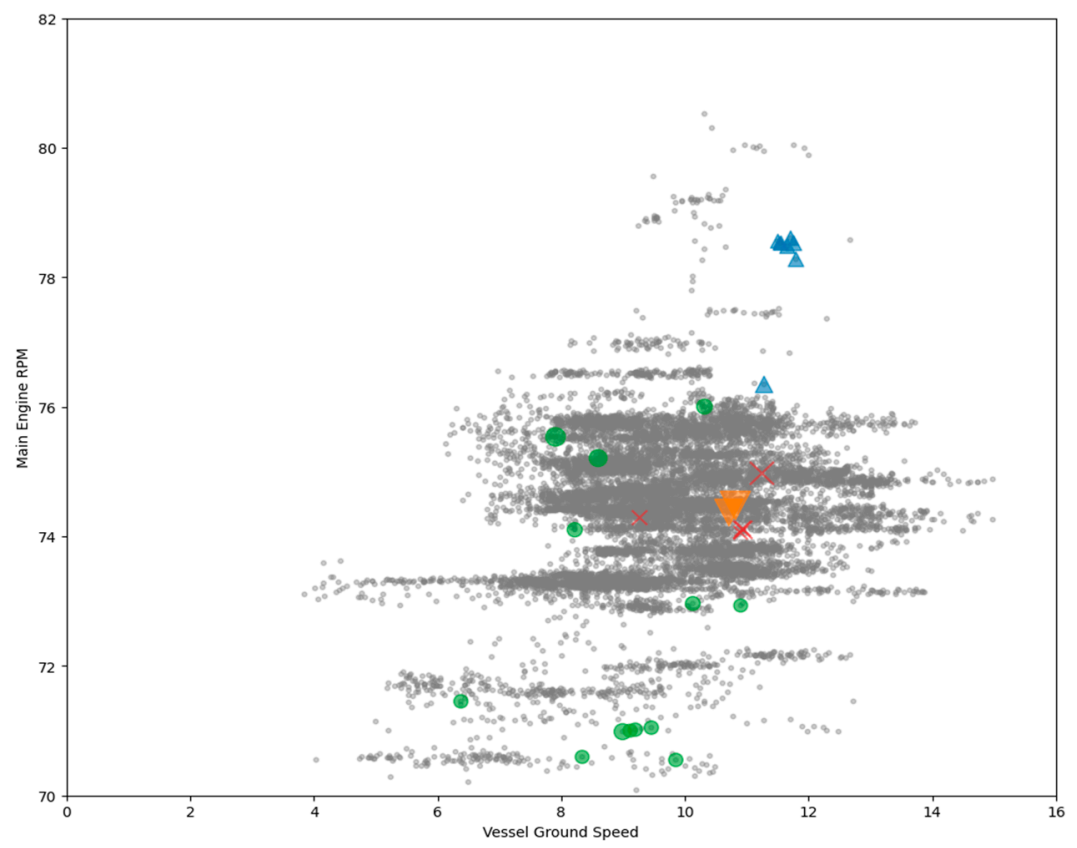

Figure 9. Anomalies plotted over speed vs. RPM scatter plot.

We also examined the anomalous data point and the time series of ground speed, as shown in Figure 10. As the figure indicated, most of the data points in cluster 1, cluster 2, and cluster 3 involved the rapid vessel speed change. This result suggests that the anomaly may be related to the acceleration or deacceleration, which may cause damage to the engine. 

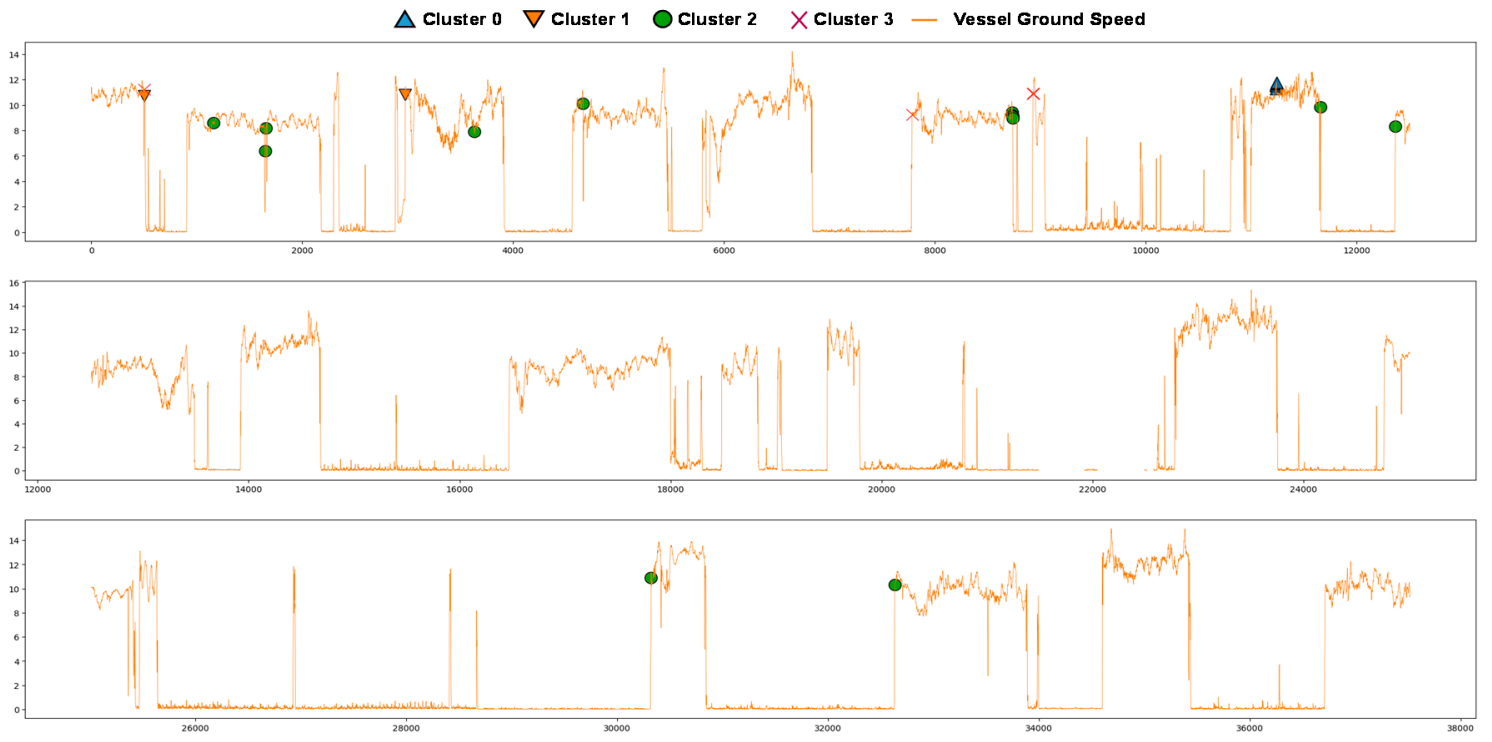

Figure 10. Anomalies plotted over the ground speed of the vessel.

Finally, we plotted the anomaly over the vessel route, as shown in Figure 11. The thin black line illustrates the navigation route of the ship for over ten months. As shown in this figure, most of the anomalies occurred in near lands, except few cases. The possible explanation for this might be that the vessel is usually driven at a low speed in the coastal waters to prevent an accident, and the engine is operated in a different pattern than usual due to frequent changes in speed. For this reason, data in the coastal waters can be classified as anomalous. We think that such information will help locate the cause of engine anomaly during ship operation in the future.

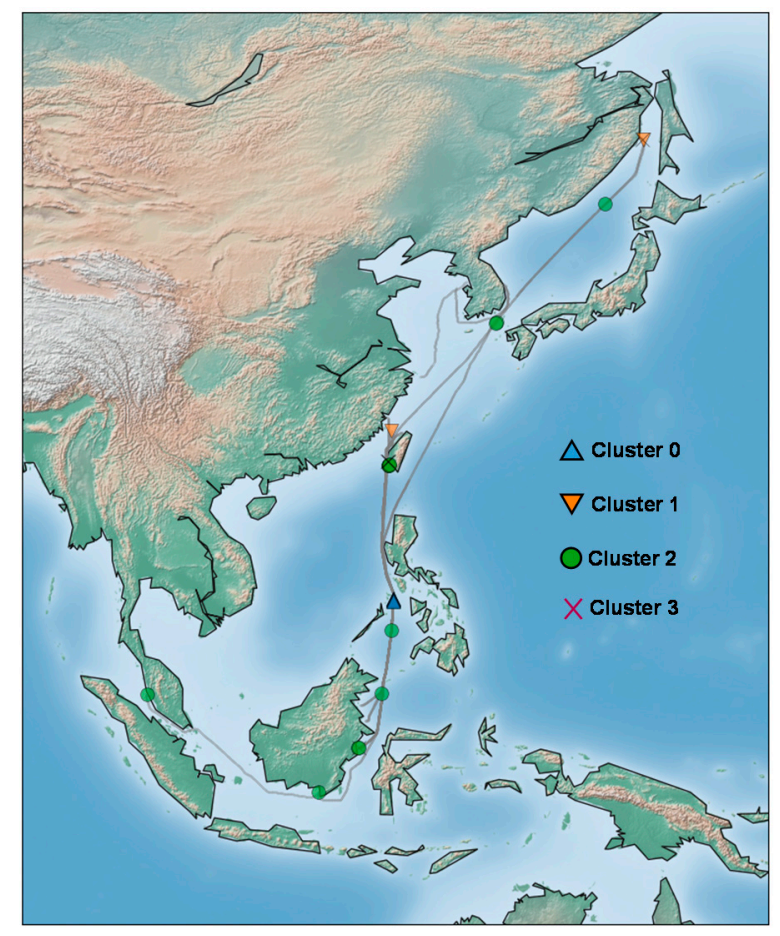

Figure 11. Anomalies plotted over the vessel routes. 


\section{Conclusions}

In this work, a machine learning approach is adopted to detect the anomalous vessel main engine. We collected an actual dataset of a large-scale bulk carrier over ten months. This study adopted an ensemble-based algorithm to learn the large-scaled and high-dimensional engine sensor streams. As a result, each data point was successfully measured with a unified measure. With this framework, one can detect the anomalous engine behavior that shows a large deviation from the normal condition. In this study, we also conducted a clustering analysis to examine the common patterns of anomalies and which can provide information for the engine diagnosis.

The limitations of our research are as follows. First, the current dataset does not include external factors, such as the seawater temperature or air temperature. Although the cooling system in our target vessel operates to control the effect of such external factors, the relationship between external factors and engine performance should be investigated in future studies. Moreover, more rigorous preprocessing may improve the analysis result. Currently, only the outlier whose sensor value is outside of measurement range were removed from the dataset. However, some outliers may be within the measurement range, but does not satisfy the physical constraint. For example, the temperature of the exhaust gases at the inlet of the turbocharger must be higher than at the outlet. Accommodating such physical condition across sensor variables could be considered in the future works. Moreover, in future studies, we need to obtain a complete dataset. We need to increase the dataset size by extending the data collection period or combining another vessel's dataset with the same engine type.

Despite the above limitation, the unsupervised approach proposed in this paper could be used for the initial screening of the engine status monitoring and can be combined with other fault diagnosis methods. One of the natural extensions of this work is to apply an existing failure mode analysis framework to the anomalies detected by our data-driven approach. There are several works for identifying possible cause and symptoms of marine engines. Those failure modes were usually obtained from the expert knowledge [33] or the simulation experiment [32]. The development of the framework and visualization scheme for relating such failure modes and the anomalies may be helpful for fault isolation and diagnosis. Our methodology can also be extended to another subsystem. The current work only analyzed the engine-related parameters. However, the modern vessel collects sensor stream from various subsystems including cargo management, or power generation system. Considering those subsystems, thus, would be a fruitful area for future works. Another possible extension of this work is to develop more efficient method for analyzing anomalies patterns. Even though clustering analysis was conducted to explain the common cause of anomalous data, it still depends on the visual inspection, making it difficult to explain the cause of anomaly quantitatively. We can improve the analysis by adopting an Explainable Artificial intelligence (EAI) framework, such as Shapley Additive Explanation (SHAP) [34] or Local Interpretable Model-agnostic Explanations (LIME) [35], which quantifies feature contribution to an individual anomalous data point. With feature importance information, more rigorous analysis for categorizing anomalous patterns by focusing on problematic sensor values may be possible.

Author Contributions: Conceptualization, D.K.; data curation, S.L.; formal analysis, J.L.; investigation, J.L.; methodology, J.L.; project administration, D.K.; supervision, J.L.; validation, J.L.; visualization, J.L.; writing - original draft, J.L. All authors have read and agreed to the published version of the manuscript.

Funding: This research was financially supported by the Ministry of Trade, Industry, and Energy (MOTIE) and Korea Institute for Advancement of Technology (KIAT) through the National Innovation Cluster R\&D program (Advancement of an open cloud platform for smart maritime convergence service_P0015306).

Conflicts of Interest: The authors declare no conflict of interest. 


\section{References}

1. Lazakis, I.; Turan, O.; Aksu, S. Increasing ship operational reliability through the implementation of a holistic maintenance management strategy. Ships Offshore Struct. 2010, 5, 337-357. [CrossRef]

2. Jiang, R.; Yan, X. Condition Monitoring of Diesel Engines. In Complex System Maintenance Handbook; Springer: London, UK, 2008.

3. Kandemir, C.; Celik, M. A human reliability assessment of marine auxiliary machinery maintenance operations under ship PMS and maintenance 4.0 concepts. Cogn. Technol. Work 2019, 22, 1-15. [CrossRef]

4. Jimenez, V.J.; Bouhmala, N.; Gausdal, A.H. Developing a predictive maintenance model for vessel machinery. J. Ocean. Eng. Sci. 2019, 5, 358-386. [CrossRef]

5. Perera, L.P.; Mo, B. Marine engine-centered data analytics for ship performance monitoring. J. Offshore Mech. Arct. Eng. 2017, 139, 021301. [CrossRef]

6. Mak, L.; Sullivan, M.; Kuczora, A.; Millan, J. Ship performance monitoring and analysis to improve fuel efficiency. In Proceedings of the 2014 Oceans-St. John's, St. John's, NL, Canada, 14-19 September 2014.

7. Aldous, L.; Smith, T.; Bucknall, R.; Thompson, P. Uncertainty analysis in ship performance monitoring. Ocean. Eng. 2015, 110, 29-38. [CrossRef]

8. Soner, O.; Akyuz, E.; Celik, M. Use of tree-based methods in ship performance monitoring under operating conditions. Ocean. Eng. 2018, 166, 302-310. [CrossRef]

9. Kim, D.; Lee, S.; Lee, J. Data-Driven Prediction of Vessel Propulsion Power Using Support Vector Regression with Onboard Measurement and Ocean Data. Sensors 2020, 20, 1588. [CrossRef] [PubMed]

10. Chi, H.; Pedrielli, G.; Ng, S.H.; Kister, T.; Bressan, S. A framework for real-time monitoring of energy efficiency of marine vessels. Energy 2018, 145, 246-260. [CrossRef]

11. Gkerekos, C.; Lazakis, I.; Theotokatos, G. Machine learning models for predicting ship main engine Fuel Oil Consumption: A comparative study. Ocean. Eng. 2019, 188, 106282. [CrossRef]

12. Strušnik, D.; Avsec, J. Artificial neural networking and fuzzy logic exergy controlling model of combined heat and power system in thermal power plant. Energy 2015, 80, 318-330. [CrossRef]

13. Strušnik, D.; Marčič, M.; Golob, M.; Hribernik, A.; Živić, M.; Avsec, J. Energy efficiency analysis of steam ejector and electric vacuum pump for a turbine condenser air extraction system based on supervised machine learning modelling. Appl. Energy 2016, 173, 386-405. [CrossRef]

14. Perera, L.P.; Mo, B. Data analysis on marine engine operating regions in relation to ship navigation. Ocean. Eng. 2016, 128, 163-172. [CrossRef]

15. Perera, L.P.; Mo, B. Marine engine operating regions under principal component analysis to evaluate ship performance and navigation behavior. IFAC-Pap. 2016, 49, 512-517. [CrossRef]

16. Brandsæter, A.; Vanem, E.; Glad, I.K. Efficient on-line anomaly detection for ship systems in operation. Expert Syst. Appl. 2019, 121, 418-437. [CrossRef]

17. Vanem, E.; Brandsæter, A. Unsupervised anomaly detection based on clustering methods and sensor data on a marine diesel engine. J. Mar. Eng. Technol. 2019, 1-18. [CrossRef]

18. Bae, Y.M.; Kim, M.J.; Kim, K.J.; Jun, C.H.; Byeon, S.S.; Park, K.M. A Case Study on the Establishment of Upper Control Limit to Detect Vessel's Main Engine Failures using Multivariate Control Chart. J. Soc. Nav. Archit. Korea 2018, 55, 505-513. [CrossRef]

19. Phaladiganon, P.; Kim, S.B.; Chen, V.C.; Baek, J.G.; Park, S.K. Bootstrap-based T 2 multivariate control charts. Commun. Stat. Simul. Comput. 2011, 40, 645-662. [CrossRef]

20. Mason, R.L.; Young, J.C. Multivariate Statistical Process Control with Industrial Applications; Society for Industrial and Applied Mathematics: Philadelphia, PA, USA, 2002.

21. MAN Two Stroke-Project Guide. Available online: https://marine.man-es.com/two-stroke/project-guides (accessed on 20 November 2020).

22. Chu, C.S.J. Time series segmentation: A sliding window approach. Inf. Sci. 1995, 85, 147-173. [CrossRef]

23. Chandola, V.; Banerjee, A.; Kumar, V. Anomaly detection: A survey. ACM Comput. Surv. 1995, 41, 1-58. [CrossRef]

24. Das, S.; Wong, W.K.; Dietterich, T.; Fern, A.; Emmott, A. Incorporating expert feedback into active anomaly discovery. In Proceedings of the 2016 IEEE 16th International Conference on Data Mining, Barcelona, Spain, 12-15 December 2016.

25. Aggarwal, C.C. Outlier ensembles: Position paper. ACM Sigkdd Explor. Newsl. 2013, 14, 49-58. [CrossRef] 
26. Breunig, M.M.; Kriegel, H.P.; Ng, R.T.; Sander, J. LOF: Identifying density-based local outliers. In Proceedings of the 2000 ACM SIGMOD International Conference on Management of Data, Dallas, TX, USA, 16-18 May 2000.

27. Ramaswamy, S.; Rastogi, R.; Shim, K. Efficient algorithms for mining outliers from large data sets. ACM Sigmod Rec. 2000, 29, 427-438. [CrossRef]

28. Aggarwal, C.C. Outlier Analysis; Springer International Publishing: Cham, Switzerland, 2017.

29. Zhao, Y.; Nasrullah, Z.; Hryniewicki, M.K.; Li, Z. LSCP: Locally selective combination in parallel outlier ensembles. In Proceedings of the 2019 SIAM International Conference on Data Mining, Calgary, AB, Canada, 2-4 May 2019.

30. Zhao, Y.; Nasrullah, Z.; Li, Z. PyOD: A Python Toolbox for Scalable Outlier Detection. J. Mach. Learn. Res. 2019, 20, 1-7.

31. Huang, Y.; Hong, G. Investigation of the effect of heated ethanol fuel on combustion and emissions of an ethanol direct injection plus gasoline port injection (EDI + GPI) engine. Energy Convers. Manag. 2019, 123, 338-347. [CrossRef]

32. Rubio, J.A.P.; Vera-García, F.; Grau, J.H.; Cámara, J.M.; Hernandez, D.A. Marine diesel engine failure simulator based on thermodynamic model. Appl. Therm. Eng. 2018, 144, 982-995. [CrossRef]

33. Cicek, K.; Turan, H.H.; Topcu, Y.I.; Searslan, M.N. Risk-based preventive maintenance planning using Failure Mode and Effect Analysis (FMEA) for marine engine systems. In Proceedings of the 2010 Second International Conference on Engineering System Management and Applications, Sharjah, UAE, 30 March-1 April 2010.

34. Lundberg, S.M.; Lee, S.I. A unified approach to interpreting model predictions. In Proceedings of the 31st International Conference on Neural Information Processing Systems, Long Beach, CA, USA, 4-9 December 2017.

35. Ribeiro, M.T.; Singh, S.; Guestrin, C. "Why should I trust you?" Explaining the predictions of any classifier. In Proceedings of the 22nd ACM SIGKDD International Conference on Knowledge Discovery and Data Mining, San Francisco, CA, USA, 13-17 August 2016.

Publisher's Note: MDPI stays neutral with regard to jurisdictional claims in published maps and institutional affiliations.

(C) 2020 by the authors. Licensee MDPI, Basel, Switzerland. This article is an open access article distributed under the terms and conditions of the Creative Commons Attribution (CC BY) license (http://creativecommons.org/licenses/by/4.0/). 